\title{
Mise en Scène: A Film Scholarship Augmented Reality Mobile Application
}

\author{
Joseph T. Chao, Tanxin Du, Christopher P. Wagenheim, \\ and Theodore F. Rippey \\ Bowling Green State Univ., Bowling Green, Ohio, USA
}

\author{
ichao@bgsu.edu tdu@bgsu.edu cwagenh@bgsu.edu \\ theodor@bgsu.edu
}

\begin{abstract}
Traditional film scholarship publication methods - an arrangement of texts and still images on printed paper - inhibit an author's ability to accurately convey the aural and kinetic nature of the medium. With information overlaid onto reality by Augmented Reality (AR) technology the gap between static text and still imagery and aural and kinetic digital media is drastically reduced. This paper describes the development of a film scholarship AR mobile application for film scholarship that provides an aural and kinetic experience for readers by allowing them to access augmented, multimedia content in real time. AR is utilized by this application for its ability to connect digitally enhanced views of the real world with meaningful content. Mise en Scène is an AR technology mobile application that allows for extra information to be augmented and displayed by tracking still images and text in academic publications. Mise en Scène was developed and implemented as a student service-learning class project following agile software development methodology.
\end{abstract}

Keywords: Augmented Reality, Mobile Application, Agile, iOS Development, Film Scholarship

\section{Introduction}

The presentation of film scholarship in the United States, like the majority of scholarship in academia, has remained relatively unchanged for decades; it was, and is, primarily textual. However, in addition to printed text, film scholarship has often employed the still frame or screen capture as a way of integrating film's visual dimension. And while the screen capture continues to be a valuable tool in the presentation of film analysis, it is only one of many possible forms of multimedia. Mise en Scène, an augmented reality (AR) mobile application, is a tool that has been designed to bring cinema scholarship into a kinetic and auditory realm more closely in line with the

Material published as part of this publication, either on-line or in print, is copyrighted by the Informing Science Institute. Permission to make digital or paper copy of part or all of these works for personal or classroom use is granted without fee provided that the copies are not made or distributed for profit or commercial advantage AND that copies 1) bear this notice in full and 2) give the full citation on the first page. It is permissible to abstract these works so long as credit is given. To copy in all other cases or to republish or to post on a server or to redistribute to lists requires specific permission and payment of a fee. Contact Publisher@InformingScience.org to request redistribution permission. actual medium being studied: film.

By embracing digital mobile technology, specifically AR, Mise en Scène is able to augment printed articles and books with short video examples, audio clips, and image galleries. Mise en Scène can augment both existing scholarship and works that are crafted with the technology specifically in mind. It should be noted that some attempts have been made to implement audio and 
video into film scholarship, but these attempts have been limited in both the technology used and the exposure achieved. Journals like Mediascape, Brighlights Film Journal, Refractory, and Underground Film Journal do embed videos into their online articles using Flash players like YouTube or JW Player, but in a rudimentary way that resemble blog articles. Additionally, the PDF versions of their articles that are available to print do not contain any ability to access the selected content.

This project was a result of frustration with the current limitations in traditional scholarly exhibition. The impetus was an unpublished article on Alfred Hitchcock's Strangers on a Train that included nearly 30 still images in a protracted appendix. Due largely in part to physical space constraints, the inclusion of more than only a few still images in a single article on film is largely discouraged within the community of publishers and editors and thus the Hitchcock article was untenable from a publishing perspective. It became apparent that the number of still images in the Hitchcock article could have been reduced had the exhibition format of scholarly findings allowed for the inclusion of video and audio clips instead of just screen captures.

Augmented reality offered two unique opportunities for film scholarship: a seamless merging of digital content and physical paper and the ability to more easily enhance archived works of nondigitized film scholarship. In the original conception of Mise en Scène as an enhanced PDF reader, film scholarship would either have to be created digitally with the application in mind or already-printed, pre-digital texts would have to be digitized and then enhanced. With augmented reality, physical books and articles could be enhanced/augmented without the need for digitization of the original work - digital and analogue would no longer have to be mutually exclusive. It is easily within the realm of possibility that a book or article from the early 20th century could, without digitization, be enhanced with digital multimedia. While this cross-generation augmentation was purely conceptual in the early stages of the project, the concept became reality when the Mise en Scène development teams were not only able to isolate specific articles to ensure there wouldn't be any overlaps between documents, but also figured out a way to instruct the AR software to recognize unique chunks of text, not just images. These breakthroughs allow any book or article from any time and any printer to be uniquely augmented with digital multimedia content without having to digitize the original source.

Mise en Scène not only addresses an enormous technological gap in the film-scholarly exchange, it accurately reflects the complexity of the medium of film; cinema scholarship, for the first time in its history, can be presented in a way that utilizes the aural and kinetic nature of film itself. Mise en Scène also represents an opportunity to reach a younger, more technologically driven audience. As technology moves towards more mobile platforms, the presence of film scholarship on these platforms is vital. Mise en Scène could potentially increase interest in film scholarship by reaching a younger academic audience on an advanced platform that they are both familiar and comfortable with. The extension of film scholarship into more culturally pervasive platforms with sonic and kinetic capabilities could create an academic experience that might stoke a new generation of film scholars.

\section{Literature Review}

Augmented reality is commonly defined as a direct or indirect view of real-time physical surroundings enhanced by adding virtual, computer-generated information onto it (Elmqvist \& Tsigas, 2008). The technology was first used in a first person indoor/outdoor AR game, ARQuake (Thomas et al., 2000), which used a wearable computer system called Tinmith (Piekarski, Gunther, \& Thomas, 1999). The Tinmith wearable system included a laptop with Linux operating system, a head-mounted display (HMD), and other sensors such as a Global Positioning System (GPS) and digital compass. ARQuake is an extension of Quake, a first person shooter computer game, which allows users to see AR content, such as monsters, weapons, and other objects of in- 
terest, in the real physical environment. AR technology has also been used outside of gaming to visualize indoor spatial information and location direction. Reitmayr and Schmalstieg (2003) built a system composed of a laptop computer running Windows operating system, a wireless network adapter, an InterSense Inter-Trax 2 orientation sensor, a web camera, and a see-through stereoscopic color HMD. In order to realize this indoor AR navigation system, they also built an indoor tracking system based on fiducial markers. However, both of these projects were restricted by the mobile computing abilities of their time. The hardware these AR technologies required were not portable enough to be convenient for comfortable carry. Fiducial markers, commonly used as triggers for AR contents, can also be complicated as they require certain maintenance.

With the new advances in mobile technologies both in hardware and software, markerless AR gradually replaced fiducial marker based AR. Markerless AR relies on natural features of reality instead of fiducial markers which are not really part of the surroundings; markerless triggers (or markers) can be directly extracted from environment characteristics. However, the markerless AR tracking (or scanning) techniques are decidedly more complex. Nevertheless, markerless AR technology has been widely used in many areas. Miyashita et al. (2008) utilized markerless tracking technology to develop an AR museum-guide application. Additionally Renukdas, Ghundiyal, Gadgil, and Pathare (2013) proposed a markerless application used for interior decoration. This application ultimately enabled users to decorate rooms by inserting virtual 3D furniture models into real environments.

Recently, AR technology has been primarily employed in two types of display technologies. The first type of display technology is innovative wearable devices that can overlay AR imagery onto a real world view via eyeglasses. Google Glass and META's Spaceglass are notable examples of this kind of display technology. The second type of display technology is handheld mobile phones. Modern smartphones are equipped with powerful CPUs, gravity sensors, integrated front and rear cameras, light sensors, GPS sensors, and touch screen interfaces, which makes them the most suitable platforms for AR software. Considering the complexity and cost of developing a wearable device, and the ubiquity of smart phones, most companies have developed their AR technology based on a universal cellphone platform.

Many mobile AR applications currently exist. Chehimi, Coulton, and Edwards (2007) were one of the first to introduce a unique AR system that allowed users to see complex and interactive visual 3D advertisements on mobile phones via its camera interface. They developed a camerabased mobile phone application on the Symbian OS platform, which was designed to locate and track QRcode in order to display 3D advertising content. Additionally, users can interact with advertising content by rotating around the augmentation as well as changing their distance from it. AR mobile phone applications have also been used for indoor navigation. Delail, Weruaga, and Zemerly (2012) created an iOS mobile application called CAViAR for indoor AR navigation. CAViAR provides indoor positioning service based on image marker recognition and inertial measurement. The AR layer provides information about nearby points of interest on the screen and displays nametags or room numbers to users. This application also used Optical Character Recognition (OCR) technology that was explored in Fragoso, Gauglitz, Zamora, Kleban, and Turk (2011). According to Fragoso's work, an AR translator was created based on Symbian platform and an OCR algorithm. The application uses the built-in camera to track real-time snapshots. The text in the snapshots can be extracted, tracked, translated, and finally displayed on the screen in real-time.

AR mobile phone applications have also been widely used in commercial settings, such as the IKEA Catalog (http://info.ikea-usa.com/Catalog), magazines, and movie posters (Layar, 2013). The most ubiquitous embrace of AR technology has been by multinational corporations as attention grabbing advertising. The most notable of these AR advertisements are located in Time Square in New York City (Mathieson, 2011). However, augmented reality has also left a rela- 
tively large impression on the art world through the construction of digital installations like those created by Yang \& Li, (n.d.). Live theatre is also experimenting with the technology by creating virtual characters (Mavridis \& Hanson, 2009) and museums are starting to create digital collections through the implementation of augmented reality (Wojciechowski, Walczak, White, \& Cellary, 2004). Not surprisingly, it is an artist/scholar that is exploring the impact of AR on film. Andrew Roth of the Humanities, Arts, Science, and Technology Alliance and Collabratory at York University in Toronto is "investigating a creative application of the use of intelligent agents in spatial narrative structures" (Roth, 2013), especially as it pertains to the future of documentary filmmaking. Perhaps the most salient example of AR relevant to Mise en Scène, however, is the work of Anomaly Productions based out of Laguna Hills California. Anomaly has produced two augmented graphic novels that provide "additional details, backstory and more than 503 -D models via an accompanying mobile app" (Schmidt, 2013). Anomaly Productions successfully bridge the divide between digital content and traditionally printed, paper material. As of now, there aren't any attempts outside of this project to augment scholarship using AR. Mise en Scène in the first of its kind in the academic community.

\section{The Project and the Process}

The project was designed to create a working prototype of an AR mobile application for film scholarship presentation that would allow readers to access augmented multimedia material while reading printed journal articles and other publications that are composed of printed text and still images. The core objective was to introduce the kinetic and sonic dimensions of cinema into the experience of film studies scholarly exchange. Mise en Scène allows the user to access augmented content in real time.

The main feature of Mise en Scène is to scan images (pictures, photographs, film stills) contained in static texts that are linked to a video clip, sound clip, other images or information, and gives the user the ability to display, listen, or watch the additional augmented content. It also recognizes text-based phrases (word combinations, sentences, and paragraphs) that are linked to additional augmented content. These images and word phrases are the "markers" that enable the document to be searched, scanned, and recognized. Mise en Scène also keeps track of user history, which includes previously viewed AR content, how the content was triggered and by which markers, in which articles, and on what date and time. Mise en Scène also provides users the ability to bookmark augmented contents for future references.

These features were established through user stories. A user story is an agile software development technique that consists of system requirements written in non-technical language that captures what the user wants. Mise en Scène had to address two types of users. They are the journal article authors who want to design and upload their AR contents to the system, and the article readers who are to use the AR app to access AR content. The list of user stories for both the authors and the readers are given in Table 1 and Table 2, respectively. 
Table 1. User Stories for Authors

\begin{tabular}{|l|l|}
\hline \multicolumn{2}{|c|}{ AS AN AUTHOR } \\
\hline \multirow{2}{*}{1} & \begin{tabular}{l} 
As an author I want to be able to upload augmented content in relation to a particular article. \\
\cline { 2 - 3 } \\
en Scène article. This content would be video, text, audio or image galleries. Readers can use \\
this content to improve their experience while reading a Mise en Scène article.
\end{tabular} \\
\hline \multirow{2}{*}{2} & $\begin{array}{l}\text { As an author I want to be able to edit/delete augmented content in relation to a particular article. } \\
\text { This would involve uploading a trigger along with associated content assigned to a given Mise } \\
\text { en Scène article. This content would be video, text, audio or image galleries. Readers can use } \\
\text { this content to improve their experience while reading a Mise en Scène article. }\end{array}$ \\
\hline
\end{tabular}

Table 2. User Stories for Readers

\begin{tabular}{|c|c|}
\hline \multicolumn{2}{|r|}{ AS A READER } \\
\hline 1 & $\begin{array}{l}\text { As a reader I want to be able to scan images in my Mise en Scène articles with a mobile device } \\
\text { camera so that I can experience the benefits of AR associated with images added to a Mise en } \\
\text { Scène Article. }\end{array}$ \\
\hline 2 & $\begin{array}{l}\text { As a reader I want to scan a Mise en Scène article and find augmented content triggers with a } \\
\text { camera on my mobile device so that I can access the associated augmented content. }\end{array}$ \\
\hline 3 & $\begin{array}{l}\text { As a reader I want to be able to access past augmented experiences, be they video, text, audio } \\
\text { or image galleries quickly so that I can re-experience augmented content even when that con- } \\
\text { tent isn't immediately in front of me. }\end{array}$ \\
\hline 4 & $\begin{array}{l}\text { As a reader I want to be able to view multimedia AR, such as video and audio clips played } \\
\text { through mobile device's local media suites so that I can easily watch clips and experience au- } \\
\text { dio being talked about in the current article I am reading. }\end{array}$ \\
\hline 5 & $\begin{array}{l}\text { As a reader I want augmented content to be scalable to the distance between mobile device } \\
\text { camera and the sheet of paper so I can see more accurately where the augmented content trig- } \\
\text { ger is located on the page. }\end{array}$ \\
\hline 6 & $\begin{array}{l}\text { As a reader I want multiple images associated with augmented content triggers to be shown in } \\
\text { an image gallery so that I can easily view all images connected to said trigger. }\end{array}$ \\
\hline 7 & $\begin{array}{l}\text { As a reader I want to be able to search for articles that have augmented content by entering in } \\
\text { an author's name, a volume name, or an article so that I do not have to struggle to look for } \\
\text { augmented content for the article currently being read. }\end{array}$ \\
\hline 8 & $\begin{array}{l}\text { As a reader I want to be able to scan a block of text with a mobile device camera and Mise en } \\
\text { Scène recognize that paragraph or string of sentences so that augmented content can be trig- } \\
\text { gered. }\end{array}$ \\
\hline 9 & $\begin{array}{l}\text { As a reader I want to be able to easily play video clips as they may appear from augmented } \\
\text { content triggers in Mise en Scène article so that I can quickly and easily experience a video clip } \\
\text { that relates to my article. }\end{array}$ \\
\hline 10 & $\begin{array}{l}\text { As a reader I want to be able to easily play audio clips as they may appear from augmented } \\
\text { content triggers in Mise en Scène article so that I can quickly and easily experience audio con- } \\
\text { tent that relates to my article. }\end{array}$ \\
\hline 11 & $\begin{array}{l}\text { As a reader I want to be able to create citations for video/audio/text/image galleries so that I } \\
\text { can share that piece of augmented content with an additional Mise en Scène user or be able to } \\
\text { record the citation for future reference. }\end{array}$ \\
\hline
\end{tabular}


This project was developed iteratively and incrementally using agile methods based on eXtreme Programming (Beck, 2000) and Scrum (Schwaber, 2009) by students in a software development class. This approach has been applied successfully in previous years by the author, and was recommended by several other educators (Alfonso \& Botia, 2005; Chao \& Brown, 2009; Davey \& Parker, 2010).

\section{The Technology}

Creating an app that give readers the ability to see augmented multimedia content when scanning with a mobile device requires two major technologies: mobile app development and augmented reality. In addition, depending on the app design, it may also require a database and an application server for information storage and retrieval.

For mobile development, the two common development approaches are 1) Create native apps targeted at some or all of the major mobile platforms (e.g. Android, iPhone, Windows, etc.), or 2) Use a web framework to create one application that can be optimized for multiple mobile platforms. The major advantage for web framework over native apps is "write once, deploy everywhere". That means, after writing the application using a web framework, the app can be packaged to run on all mobile platforms that are supported by the framework. In addition, most developers are already familiar with web technologies and therefore the learning curve is usually not as steep. However, native applications can perform more efficiently on a mobile OS and could use many native functions provided by the mobile OS. Native user interface (UI) characteristics could be considered in UI design for native mobile applications which may comply with users' habits. A web application framework uses one-size-fits-all design, which may result in a longer learning curve or degrade the ease of use experience for end-users.

Since user experience and app performance was a high priority requirement, the decision was made to develop Mise en Scène as a native mobile app. Once the native app development decision was made, a mobile platform had to be selected to start the development. While there are many platforms to choose from, the two most popular choices are iOS and Android. The iOS system runs on Apple mobile devices including iPhone, iPod, and iPad, while the Android system offers a considerably open platform for developers. However, compatibility problems on Android were a major concern due to the various models of smartphones running different versions of Android operating systems. The iOS system was ultimately chosen as the platform for the application due to its consistency. A native integrated development environment from Apple, XCODE including iOS Software Development Kit (SDK), was used for development.

Several AR SDKs, needed to help Mise en Scène realize the required AR functionality, were available at the time of development. We evaluated four major AR SDKs - ARToolkit, Metaio SDK, Vuforia, and Layar - according to the following criteria:

1. Mobile platform compatibility,

2. Required AR features,

3. Reasonable financial cost, and

4. Availability of support.

All four AR SDKs are compatible with both iOS and Android mobile platforms, and contain the required features (including marker tracking, image tracking, and visual search) for Mise en Scène. The decision came down to the cost and the availability of support. Financial cost was somewhat complicated since most of the fee structures are based on usage limitation, and therefore highly dependent on the type of application being developed. However, all of the SDKs offered free trial versions, which was good enough for developing proof of concept systems. The final decision came down to the availability of support, and it was decided that Metaio SDK pro- 
vided the most complete set of tutorials and examples, and that it also offered an excellent technical support forum which is most helpful for students in regards to flattening their learning curve.

AR workflow involved two steps: 1) tracking/recognizing objects (markers) from a reality view; 2) showing augmented content corresponding to the tracking result. By using Metaio SDK, these steps could be done in the background. When the application is running, the image that the user scans is compared with AR triggers located on the mobile device. Once the image and one of the AR triggers have similarity at a pre-set percentage, Metaio SDK will map the tracked AR marker to associated content and show it on the screen in real time.

\section{The Design and Implementation}

Mise en Scène is based on a client-server architecture design. The mobile phone app acts as the client, and an application server, which is both a data server and the server-side web application that allows administrative users to manage augmented contents for journal articles, constitutes the server portion. The system may start with an administrative user, via the server-side web application, uploading a tracking image with its associated augmented content, which could be a video, an audio clip, a picture, an image gallery, or a textual document for a particular article. The administrative user may upload augmented content for as many articles as they wish, and each article may contain multiple tracking images designed to be scanned by journal article readers. A database located on the app server is designed for storing the tracking images and their corresponding augmented content. Once the tracking files and augmented content have been uploaded into the database, the client-side mobile app is ready to be used.



Figure 2. Mise en Scène User Interface

The mobile app on the client device will first ask the user, a journal article reader, to indicate which article to scan for augmented content by selecting a journal volume, issue, and article title from a list. An example is given in Figure 2. As soon as an article is specified by the user, the mobile app will send a request to the app server, and the tracking images for the article will be downloaded so that the user can scan the printed article with the camera on the mobile device searching for the tracking images. When a tracking image is found on the article, the app will display the trigger image, such as a play button as shown in Figure 3, which allows the user to click and view the corresponding augmented content. 




Figure 3. Mise en Scène User Interface

One requirement when using Metaio SDK to scan articles for tracking images (the markers), is that the tracking image files must be located on the same device as the AR mobile app. One original design was to download all tracking image files for all articles to the mobile device to facilitate fast scanning on all articles for augmented contents. However, the large number of articles in film journals published in past decades rendered this approach impractical due to the limited storage on a mobile device. It also created a challenge in regards to when and how to keep the tracking files and augmented content on the mobile device up to date when the admin users made changes. Another previously considered design was to implement the scanning functionality using the "visual search" cloud service provided by Metaio. Unfortunately, the Metaio visual search service charges a monthly fee when the mobile app wants to track more than 100 objects.

The final data flow design for Mise en Scène is given in Figure 4. The tracking files and augmented contents are first uploaded to the app server. The journal reader uses Mise en Scène to select an article to scan. Mise en Scène retrieves and downloads the tracking files from the app server and stores them in a temporary folder on the mobile device. Mise en Scène scans the article for the tracking images and allows the reader to access augmented content on the app server. At the end, Mise en Scène closes, and the tracking files are deleted from the temporary folder automatically.

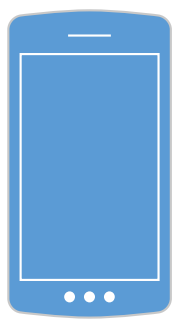

Mobile Device

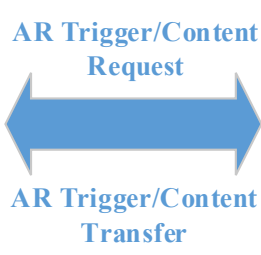



Server

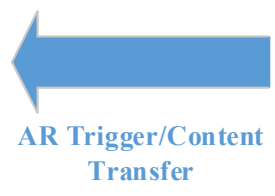

Transfer

Figure 4. Mise en Scène Data Flow Design



Database 


\section{Future Development}

At the time of writing this paper, a proof of concept version of Mise en Scène mobile app has been completed, although some originally planned features were postponed for future improvement. The most notable unfinished features include allowing article authors to upload/edit/delete augmented media content and allowing article readers to scan AR content associated with textbased phrases. These features are scheduled to be completed in the next few months. However, text recognition was alternatively and partially realized by using image tracking technology the application can recognize the shape of paragraphs within static paper articles. Although a workable solution, true text recognition would allow Mise en Scène to be more versatile.

The envisioned goal for this project, and Mise en Scène as a mobile application, is to provide text-based film journals with a tool that can seamlessly integrate digital materials into their publications. These augmented journals would, in turn, give film scholars the opportunity to write materials without limitations - authors would be free to use the full range of multimedia, including sound and video, in the creation of their articles. The authors of Mise en Scène are, at the writing of this article, collaborating with a yearly, auteur film journal, and its co-editors, to augment archived issues as further proof of concept. A more established journal-developer relationship and publicly available augmentation of current journals are dependent on the result of copyright and fair-use research, which is ongoing.

Mise en Scène developers have been, for the life of the application, a part of a digital humanities learning community comprised of several faculty members from a number of different field across the humanities. These colleagues have all expressed interest in a version of Mise en Scène that would compliment their individual disciplines. It is easy to envision Mise en Scène expanding beyond the borders of film scholarship and into virtually any realm of academic scholarship. The ability to augment text-based scholarship with digital images and sound goes beyond cinema studies and has proven to be enticing to a host of different scholars in a host of different disciplines.

\section{Conclusion}

This paper has described a film scholarship AR mobile application that utilizes AR technology to enhance reading experience for readers by letting them access augmented images, audio, and video clips in real time. Mise en Scène was developed by students enrolled in a software development class over the course of a regular semester. They followed agile software methodologies.

The technical development of Mise en Scène was informed by the selection of the iOS platform and XCODE, which was selected as the native integrated development environment. The Mise en Scène developers ultimately selected the Metaio SDK based on its compatibility, features, reasonable financial cost, and the availability of support. A client-server architecture design was determined for Mise en Scène after careful consideration. Readers can use the client application to experience augmented content, while administrative users could use the web application to manage and upload augmented triggers and associated contents. With the support of Metaio SDK, major AR features were implemented in the mobile application. Mise en Scène represents a step forward in bridging the gap between analog, text-based scholarship presentation methods and digital multimedia content in the academic realm. Not only will Mise en Scène allow film scholars to utilize the aural and kinetic aspects of the medium which they examine, it may be able to connect to students on a level that they are more familiar with. There is a serious lack of AR technology in academia. Mise en Scène addresses that gap. 


\section{References}

Alfonso, M. I., \& Botia, A. (2005). An iterative and agile process model for teaching software engineering. Proceedings of the 18th Conference on Software Engineering Education \& Training (pp. 9-16). Washington, DC, USA: IEEE Computer Society. doi:10.1109/CSEET.2005.5

Beck, K. (2000). Extreme programming explained: Embrace change. Addison-Wesley Professional.

Chao, J. T., \& Brown, J. K. (2009). Empowering students and the community through agile software development service-learning. In P. Abrahamsson, M. Marchesi, \& F. Maurer (Eds.), Agile processes in software engineering and extreme programming (pp. 104-113). Springer Berlin Heidelberg. Retrieved from http://link.springer.com/chapter/10.1007/978-3-642-01853-4_13

Chehimi, F., Coulton, P., \& Edwards, R. (2007). Augmented reality 3D interactive advertisements on smartphones. Mobile Business, International Conference on (Vol. 0, p. 21). Los Alamitos, CA, USA: IEEE Computer Society. doi:10.1109/ICMB.2007.20

Davey, W., \& Parker, K. (2010). Technology in education: An agile systems approach (pp. 297-306). Proceedings of Informing Science \& IT Education Conference (InSITE) 2010, Cassino, Italy, Informing Science Institute. Retrieved from http://researchbank.rmit.edu.au/view/rmit:15310

Delail, B. A., Weruaga, L., \& Zemerly, M. J. (2012). CAViAR: Context aware visual indoor augmented reality for a university campus. In 2012 IEEE/WIC/ACM International Conferences on Web Intelligence and Intelligent Agent Technology (WI-IAT) (Vol. 3, pp. 286-290). doi:10.1109/WI-IAT.2012.99

Elmqvist, N., \& Tsigas, P. (2008). A taxonomy of 3D occlusion management for visualization. IEEE Transactions on Visualization and Computer Graphics, 14(5), 1095-1109.

Fragoso, V., Gauglitz, S., Zamora, S., Kleban, J., \& Turk, M. (2011). TranslatAR: A mobile augmented reality translator. In 2011 IEEE Workshop on Applications of Computer Vision (WACV) (pp. 497-502). doi:10.1109/WACV.2011.5711545

Layar. (2013). Retrieved November 12, 2013, from https://www.layar.com/augmented-reality/inspiration/

Mathieson, R. (2011). Disney characters augment reality in times square promo [Video]. Retrieved December 12, 2013, from http://mathieson.typepad.com/genwow/2011/11/disney-characters-get-augmentedin-times-square-promo-video.html

Mavridis, N., \& Hanson, D. (2009). The IbnSina Center: An augmented reality theater with intelligent robotic and virtual characters. The 18th IEEE International Symposium on Robot and Human Interactive Communication, 2009. RO-MAN 2009 (pp. 681-686).

Miyashita, T., Meier, P., Tachikawa, T., Orlic, S., Eble, T., Scholz, V., ... Lieberknecht, S. (2008). An augmented reality museum guide. 7th IEEE/ACM International Symposium on Mixed and Augmented Reality, 2008. ISMAR 2008 (pp. 103-106). doi:10.1109/ISMAR.2008.4637334

Piekarski, W., Gunther, B., \& Thomas, B. (1999). Integrating virtual and augmented realities in an outdoor application. 2nd IEEE and ACM International Workshop on Augmented Reality, 1999. (IWAR '99) Proceedings (pp. 45-54). doi:10.1109/IWAR.1999.803805

Reitmayr, G., \& Schmalstieg, D. (2003). Location based applications for mobile augmented reality. Proceedings of the Fourth Australasian User Interface Conference on User Interfaces 2003 - Volume 18 (pp. 65-73). Darlinghurst, Australia, Australia: Australian Computer Society, Inc. Retrieved from http://dl.acm.org/citation.cfm?id=820086.820103

Renukdas, P., Ghundiyal, R., Gadgil, H., \& Pathare, V. (2013). Markerless augmented reality Android app for interior decoration. International Journal of Engineering, 2(4). Retrieved from http://www.ijert.org/browse/volume-2-2013/april-2013-edition?download=3110\%3Amarkerlessaugmented-reality-android-app-for-interior-decoration-\&start $=210$

Roth, A. (2013). Augmented reality, documentary, locative media. HASTAC. Retrieved December 12, 2013, from http://www.hastac.org/blogs/andrew-roth/2012/10/02/augmented-reality-documentarylocative-media 
Schmidt, G. (2013). Shifter: A graphic novel with teeth (and claws). The Standard. Retrieved December 12, 2013, from http://www.standard.net.au/story/1928242/shifter-a-graphic-novel-with-teeth-and-claws/

Schwaber, K. (2009). Agile project management with Scrum. Microsoft Press.

Thomas, B., Close, B., Donoghue, J., Squires, J., De Bondi, P., Morris, M., \& Piekarski, W. (2000). ARQuake: An Outdoor/Indoor Augmented Reality First Person Application. In Proceedings of the IEEE International Symposium on Wearable Computers (pp. 139-146).

Wojciechowski, R., Walczak, K., White, M., \& Cellary, W. (2004). Building virtual and augmented reality museum exhibitions. Proceedings of the Ninth International Conference on $3 D$ Web Technology (pp. 135-144). New York, NY, USA: ACM.

Yang, L., \& Li, H. (n.d.). Artist biography. Retrieved December 12, 2013, from http://lilyhonglei.com/courses/bio.htm

\section{Biographies}

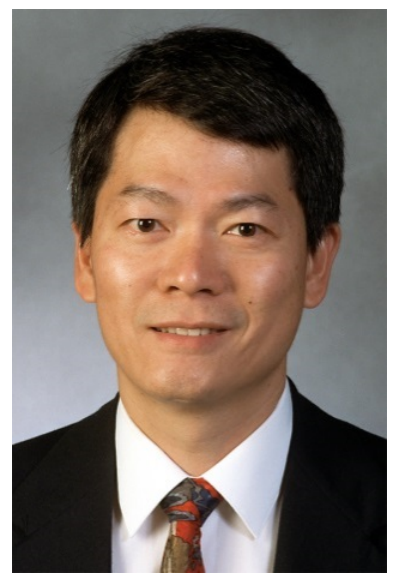

Dr. Joseph T. Chao is an Associate Professor and the Director of the Agile Software Factory in the Department of Computer Science at Bowling Green State University. The Software Factory, which he founded in 2008 with a grant from the Agile Alliance, provides students with service-learning opportunities and real-world project experience in software engineering. Prior to entering academia, Dr. Chao gained more than seven years of industry experience working as Software Engineer, System Analyst, QA Tester, Project Manager as well as Director of Software Development. His research focus is on software engineering with special interests in agile methods, and web and mobile technologies. Dr. Chao holds an M.S. in Operations Research from Case Western Reserve University and a Ph.D. in Industrial and Systems Engineering from The Ohio State University.

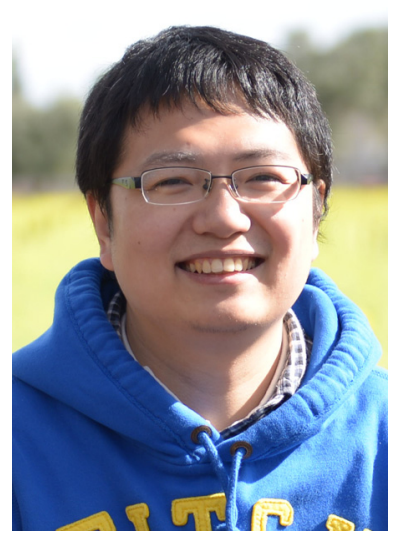

Tanxin Du is a graduate student in the Department of Computer Science at Bowling Green State University. He has also received his first Master of Science degree in Chemistry in 2012. He has focused his research on web application and iOS application development since fall 2012. Mr. Du is expected to graduate in May 2014 with a Master of Science degree in Computer Science and start his career as a software engineer. 


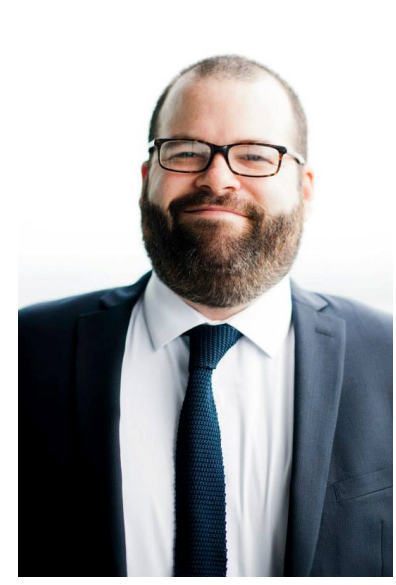

Christopher Paul Wagenheim is a doctoral candidate in American Culture Studies at Bowling Green State University in Bowling Green Ohio. His main area of research is popular American film. Wagenheim is currently writing his dissertation on the male body and masculinity in action films from the $1980 \mathrm{~s}, 1990 \mathrm{~s}$, and the $21^{\text {st }}$ century. He is also working with Dr. Joseph Chao, Dr. Theodor Rippey, and Tanxin Du on Mise en Scène, an augmented reality mobile application designed to introduce digital media into traditionally printed film scholarship. Wagenheim holds an M.A. in American Studies from the University of South Florida in Tampa.

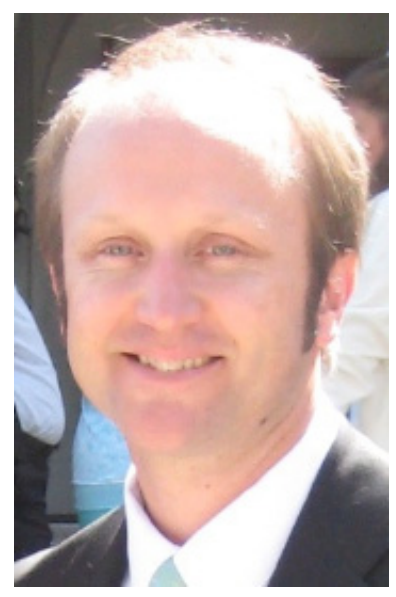

Theodore F. Rippey ( $\mathrm{PhD}$, University of Wisconsin) is Associate Professor of German and a faculty affiliate in American Culture Studies at Bowling Green State University. He has published on the cinema and literature of the Weimar Republic, the work of German writers and filmmakers exiled during the Third Reich, and the relationship of sound, media, and culture in Germany between the two World Wars. His participation in the Mise-en-Scéne project stems from his interest in the evolution of film scholarship in the emerging era of digital humanities. 